\title{
La lexicografía vasco-románica del siglo XIX: el Vocabulario vasco-castellano de Eugenio de Aranzábal*
}

\author{
NEREA FERNÁNDEZ DE GOBEO DÍAZ DE DURANA \\ Universidad Complutense de Madrid
}

\section{INTRODUCCIÓN}

Eugenio de Aranzábal, maestro de la escuela pública de la localidad vizcaína de Amorebieta, sabía de primera mano los problemas que conllevaba el desconocimiento de la lengua castellana por parte del alumnado vasco decimonónico que vivía en un entorno rural, en el que la lengua mayoritaria seguía siendo el euskera ${ }^{1}$. Esto ralentizaba el aprendizaje de los niños, puesto que la lengua en la que se impartía la enseñanza era el castellano, e impedía un progreso adecuado. Además, era consciente de la posibilidad de que hubiera profesores llegados de otros lugares que no conocieran la lengua vasca, y que fueran incapaces de poder comunicarse adecuadamente con sus alumnos.

Por todo ello, publica en 1883 el Método práctico de enseñar el castellano a los ninos vascongados en las escuelas de Vizcaya, una breve obra en la que incluye un vocabulario temático en euskera y castellano y unos ejercicios de declinación vasca y de conjugaciones de varios verbos, con el propósito de que, por un lado, los niños aprendan la lengua española con facilidad, y por otro, los maestros que no conocen el vasco puedan estudiarlo para hablar con su alumnado. Se desconoce su repercusión en las escuelas vizcaínas, porque no se han hallado ediciones posteriores; no obstante, sí que debió de ser utilizado, ya que el Método aparece citado en la Real Orden del 20 de diciembre de 1886. En ella, se presenta una lista, marcada con el número 18, que constituye la «Relación de obras declaradas útiles para que puedan servir de texto en los establecimientos de primera enseñanza, por la Comisión especial del Consejo de Instrucción pública en sesiones de 25 de Octubre y 8 de Noviembre de 1886» (Ministerio de Fomento 1887) ${ }^{2}$.

${ }^{*}$ Este trabajo se ha realizado gracias a la beca del Programa para la Formación de Profesorado Universitario, MECD (FPU-2012) y se integra dentro de los elaborados en el proyecto Creación y desarrollo de la Biblioteca Virtual de la Filología Española (FFI2011-24107), dirigido por Manuel Alvar Ezquerra.

${ }^{1}$ En el siglo XVIII, el uso del castellano es predominante en la mayor parte de Álava y en el área vizcaína de las Encartaciones (Echenique 1987: 101). Durante este siglo, se produce además una progresiva castellanización de los grandes núcleos urbanos, como Bilbao o Pamplona, que conlleva la desaparición de la lengua vasca en estos lugares en la centuria posterior, de la misma forma que ya había ocurrido en Vitoria en el siglo XVI. En los núcleos rurales vizcaínos, sin embargo, la lengua vehicular continúa siendo el vasco.

${ }^{2}$ La obra de Aranzábal se encuentra recogida en la posición número 71.

$$
\begin{array}{r}
\text { NEREA FERNÁNDEZ DE GOBEO DÍAZ DE DURANA, } \\
\text { «La lexicografía vasco románica del siglo XIX: } \\
\text { el Vocabulario vasco-castellano de Eugenio de Aranzábal», } \\
\text { Revista de Lexicografía, XX (2014), pp. 19-33 } \\
\text { Fecha de presentación: } 21 / 07 / 2014 \\
\text { Fecha de aceptación: } 12 / 03 / 2015
\end{array}
$$
ISSN: 1134-4539, e-ISSN: 2603-667. DOI: https://doi.org/10.17979/rlex.2014.20.0.3872 


\section{EL MÉTODO PRÁCTICO DE ENSEÑAR EL CASTELLANO A LOS NIÑOS VASCONGADOS EN LAS ESCUELAS DE VIZCAYA}

La obra de Eugenio de Aranzábal, impresa en Bilbao en la librería de Segundo Salvador en 1883, consta de 120 páginas y está dividida en tres apartados: un vocabulario vasco-castellano, unos ejercicios prácticos sobre declinación y un último capítulo con las conjugaciones de varios verbos. Estas secciones están precedidas por un prólogo y una explicación sobre el procedimiento que se debe seguir para la enseñanza. Al final del libro se insertan dos textos, uno en castellano y otro en euskera, para practicar la traducción. La estructura de esta obra no es original, ya que es muy similar a la que nos encontramos en una obra anterior, el Método práctico de enseñar el castellano en las escuelas vascongadas de Juan María de Eguren $(1867)^{3}$, texto concebido igualmente para la enseñanza del español al alumnado vasco.

En el prólogo, Aranzábal comenta las dificultades que hallan los niños vascos en las escuelas, en las que deben aprender las materias en lengua castellana, debido a que su lengua materna es el euskera. Como consecuencia de esto, a los jóvenes les cuesta comprender lo que leen y estudian, por lo que «el desarrollo intelectual es muy lento en ello y la enseñanza popular no progresa como debiera» (Aranzábal 1883: 4). Por tanto, la necesidad de enseñar la lengua española en estos centros es fundamental si se quiere avanzar en la educación. Sin embargo, Aranzábal comenta los problemas de algunos maestros para encontrar un método adecuado con el que enseñar el castellano, y para poder entenderse con los sus alumnos, ya que algunos de ellos no conocen el euskera:

$$
\begin{aligned}
& \text { [...] Se han visto muchos Maestros en la imposibilidad de enseñar el castellano por } \\
& \text { ignorar qué método o procedimiento debían emplear en su enseñanza, y tal vez al- } \\
& \text { gunos por no saber el vascuence, siendo por este motivo estos últimos los que más } \\
& \text { tienen que trabajar en su ministerio, sucediendo no pocas veces el no poder enten- } \\
& \text { derse con los discípulos. (Aranzábal 1883: 4) }
\end{aligned}
$$

Con la intención de resolver los problemas que ha podido advertir, el maestro escribe su Método, que puede servir de ayuda tanto a los alumnos como a los profesores de las escuelas vizcaínas.

Aranzábal realiza un resumen de lo que ya había expuesto Eguren en su prólogo, reproduciendo casi textualmente algunos fragmentos. Introduce, empero, una innova-

\begin{tabular}{|c|c|}
\hline EGUREN (1867: V-VI) & ARANZÁBAL (1883: 3-4) \\
\hline $\begin{array}{l}\text { La inmensa mayoría de los niños que asisten } \\
\text { a las escuelas de nuestro país no entiende el } \\
\text { castellano, pues que en los pueblos rurales } \\
\text { que son los que forman la mayor parte de la }\end{array}$ & $\begin{array}{l}\text { Sabido es que en los pueblos rurales, que } \\
\text { son los que forman la mayor parte de la } \\
\text { provincia de Vizcaya, solamente se habla el } \\
\text { vascuence, con muy cortas excepciones, y }\end{array}$ \\
\hline
\end{tabular}
ción que no hallamos en el método Eguren: su obra, aparte de ser utilizada por los alumnos para estudiar español, puede servir para que los maestros que no conozcan el euskera puedan aprenderlo. Aun así, la copia es evidente, como se observa en la fracción de las dos obras que se inserta a continuación:

\footnotetext{
${ }^{3}$ Para una completa descripción de la obra de Juan María de Eguren, véase García Aranda (2009).
} 
hermandad guipuzcoana, solamente se habla el vascuence, con muy cortas escepciones, y aún en las poblaciones de más importancia no es todavía habitual la lengua castellana sino entre las personas cultas y de más instrucción. Así es que la masa general del pueblo guipuzcoano habla usualmente el vascuence y por consiguiente esta es la primera lengua que aprenden los niños.

Resulta, pues, que cuando empiezan a asistir a la escuela no entienden bien el castellano, $\mathrm{y}$ como en este idioma aprenden a leer, escribir y todos los demás ramos de la enseñanza, excepto la doctrina cristiana, no comprenden lo que leen y estudian [...]. esta es, por consiguiente, la primera lengua que aprenden los niños. Cuando empiezan a asistir a la escuela, no entienden bien el castellano; y como en este idioma aprenden a leer, escribir y todo los demás ramos de la enseñanza, a excepción de la Doctrina Cristiana, que muchos de ellos estudian en vascuence, no comprenden lo que leen y estudian, como tampoco las explicaciones que les da el Maestro [...].

En cuanto al procedimiento que deben utilizar los maestros para la enseñanza del castellano en sus escuelas, Aranzábal sigue, naturalmente, el «método Eguren» ${ }^{4}$. Propone dedicar diariamente un tiempo al estudio del castellano en las escuelas, teniendo en cuenta las características propias de cada localidad:

En los pueblos donde el castellano es poco usual y conocido, se tendrá que dedicar más tiempo que en aquellos donde por su situación, industria, etc., tienen más comunicación con los que hablan castellano. Por esta razón solo el Maestro puede apreciar el tiempo que conviene invertir en estos ejercicios, teniendo en cuenta las circunstancias especiales de la localidad en que vive ${ }^{5}$. (Aranzábal 1883: 7)

De forma similar a la que encontramos en el método Eguren, Aranzábal recomienda dividir a los niños en tres secciones, y estas en grupos de no más de diez alumnos. Delante de cada uno de estos grupos se debe situar el maestro, con un ejemplar de la obra, y debe pronunciar las voces del vocabulario, por conjuntos de diez o doce palabras, primero en euskera y luego en castellano, haciendo que los niños las repitan. Tras haber aprendido de memoria todas las palabras del vocabulario, se debe pasar a la se-

${ }^{4}$ Se utiliza el término empleado por García Aranda (2009). El título de este capítulo en Aranzábal, «Procedimiento que conviene seguir en las escuelas para enseñar el castellano a los niños», es casi idéntico al de Eguren («Procedimiento que conviene seguir en las escuelas vascongadas para enseñar el castellano a los niños, y uso que al efecto debe hacerse de este libro»). El comienzo del capítulo, así como el título («Procedimiento para enseñar el castellano en las escuelas vascongadas») de la edición del Método de Eguren de 1876 son diferentes, por lo que Aranzábal se basó probablemente en la primera edición, la de 1867.

${ }^{5}$ Una vez más, copiado y resumido del texto de Eguren (1867: XI-XII): «Bien podrá suceder que en unas localidades se necesite dedicar más tiempo que en otras a este objeto, porque en pueblos rurales, compuestos de caseríos más o menos aislados y donde hay poca calle, como vulgarmente se dice en el país, el castellano es mucho menos usual y conocido que en pueblos de más importancia que, por su situación, industria de que viven sus moradores y otras circunstancias, tienen más activa y frecuente comunicación con los que hablan este idioma. Por esta razón solo el maestro, teniendo en cuenta las circunstancias especiales de la localidad en que vive, puede apreciar con exactitud el tiempo que conviene invertir en los ejercicios que vamos a reseñar $[\ldots] »$. 
gunda parte a realizar los ejercicios prácticos de declinación, sin olvidar repasar de vez en cuando el vocabulario de la primera mediante los ejercicios propuestos:

Conviene, mientras los niños permanezcan en esta segunda sección en la práctica de las declinaciones, se destine una o dos clases de la semana a los ejercicios de la primera sección para que repasen lo que aprendieron del diccionario. (Aranzábal 1883: 9)

Tras estudiar las declinaciones vascas y conocer las equivalencias en castellano, los niños han de aprender las conjugaciones de los verbos, que constituyen «una de las partes más importantes de la oración». Finalmente, se presentan dos textos, para que los niños mejoren su castellano y sean capaces de llevar a cabo traducciones de vasco a español y viceversa ${ }^{6}$.

\section{EL VOCABULARIO VASCO-CASTELLANO}

La primera parte del método de Aranzábal está constituida por un vocabulario en vasco y español ordenado temáticamente, y que recoge las voces más usuales del idioma, sin definiciones, ordenadas en diferentes epígrafes atendiendo a la realidad extralingüística. La brevedad de este vocabulario y su carácter didáctico son, junto con las anteriores, características comunes de las los repertorios léxicos conocidos como nomenclaturas, que han sido tratados de forma extensa por investigadores como Alvar Ezquerra (1987; 2002; 20137), Ayala Castro (1992) o García Aranda (2010; 2011a; 2011b).

Las nomenclaturas han sido utilizadas como medio para la enseñanza de lenguas, primero del latín y más tarde, sobre todo desde el siglo VI, para lenguas modernas (Alvar Ezquerra 2013: 19; Azorín Fernández 2000: 83). En el ámbito hispánico, las nomenclaturas empiezan a surgir con Nebrija, y su número va incrementándose a lo largo de los siglos posteriores. Muchos de estos repertorios se insertaban dentro de obras más extensas, como gramáticas o manuales para el aprendizaje de segundas lenguas, y su mayor apogeo se produce a finales del siglo XVIII y, principalmente, en el XIX:

Cuando se consolidan los métodos para la enseñanza de segundas lenguas, a finales del siglo XVIII, y, sobre todo, en el siglo XIX, a la par que se inicia su enseñanza regulada en los centros educativos, se fija también la forma y el contenido de las nomenclaturas que acompañaban a los manuales de la materia, lo cual no quiere decir que no puedan presentar diferencias entre ellas [...]. En esa época adquiere una importancia capital el Arte de Pierre Nicolas Chantreau, que será profusamente copiado e imitado, pero no será el único. Son tantas las nomenclaturas que aparecen acompañando a los manuales de enseñanza de lenguas, que, irremediablemente, unas recuerdan a las otras cuando no las copian directamente. (Alvar Ezquerra 2013: 23)

\footnotetext{
${ }^{6}$ En la obra de Eguren, en cambio, se presenta una cuarta parte con unas frases breves y unos textos para que se realice la traducción. No obstante, se recomiendan tanto la traducción al vasco del Catecismo de la Doctrina Cristiana del Padre Astete como la Historia Sagrada del Abad Fleury (Eguren 1867: XIX-XX).

${ }^{7}$ En esta obra, Alvar Ezquerra ha realizado un completo catálogo de las nomenclaturas del español desde el siglo XV hasta el XIX.
} 
Es asimismo en el siglo XIX cuando se componen por primera vez las nomenclaturas en las que aparece la lengua vasca, como la que se halla dentro de la mencionada obra de Juan María de Eguren, pero también otras conocidas como las de Luis de Astigarraga y Ugarte, Francisco Jáuregui de San Juan y Frère Juvenal Martyr ${ }^{8}$, así como la de Eugenio de Aranzábal. Estos repertorios son relevantes, puesto que la presencia del vasco en las nomenclaturas es muy poco frecuente; sin embargo, no manifiestan grandes novedades: como ha observado García Aranda (2011a), los vocabularios de Astigarraga y de Jáuregui son meras reproducciones de la nomenclatura que se encuentra dentro del Arte de Pierre Nicolas Chantreau.

\section{ESTRUCTURA Y CONTENIDO}

El repertorio de Aranzábal está dividido por categorías gramaticales, y no solo distinguen sustantivos, adjetivos y verbos, partición habitual en este tipo de obras ${ }^{9}$, sino que también separa en diferentes epígrafes participios, adverbios, preposiciones y conjunciones. Estos son similares a los utilizados por Astigarraga en su Diccionario manual, que «están a mitad de camino entre lo estrictamente léxico y lo gramatical» (Alvar Ezquerra 2013: 460).

La nomenclatura se presenta en dos columnas, la primera en vasco y la segunda en español, ya que está configurada para la enseñanza de esta lengua. Las entradas de la columna castellana son, en su mayoría, simples, aunque hay casos en los que aparecen matizaciones como «bizarra, la barba (pelo)» o «artoa, borona (pan de maíz)», adjetivos («salda garbia, el caldo limpio»; «perrechicuac setas mayores»), construcciones preposicionales («bazcaria, la comida del mediodía»; «alcondara, la camisa de hombre») o aposiciones como «lepoa, el cuello, el pescuezo»; «senarra el marido, el esposo»; «ollagorra la sorda o becada»; «bacallua el bacalao, abadejo». En la columna de la lengua vasca también encontramos aposiciones («bernea, zacoa, la pantorrilla»; «circilluac, arrecadac, los pendientes»; «bildotza, archoa, el cordero»), para una mayor caracterización de la voz, pero también como consecuencia de la variación dialectal del euskera ${ }^{10}$.

${ }^{8}$ Diccionario manual bascongado y castellano, y elementos de gramática de Astigarraga y Ugarte (1825); el vocabulario inserto en la obra Gramera berria, ikasteko eskualdunec mintzatzen espainoles de Jáuregui de San Juan (1850); y el Vocabulaire trilingue français-espagnol-basque de Juvenal Martyr (1899). Hallamos una caracterización de estos textos en García Aranda (2011b) y Alvar Ezquerra (2013).

9 Esta división «se inicia tenuemente en las nomenclaturas de Stephan Barnabé y Matthias Kramer, y se va desarrollando y afirmando en las de John Andree, Joaquín de San Pedro o Esteban de Rosterre (Terreros)» (Alvar Ezquerra 2013: 365). Ayala Castro (1992: 443) comenta que «durante los siglos XVI y XVII sólo recogían sustantivos, en el siglo XVIII entraron los verbos, en el XIX, época de aparición de nuestros diccionarios enciclopédicos, los nombres propios».

${ }^{10}$ Se hace referencia a esta variación geográfica en la «Advertencia» que se inserta al comienzo del vocabulario vasco-castellano:

En este vocabulario podrá suceder que se encuentren voces, aunque pocas, que en la localidad en que se hace uso de él se digan de otra manera, pues a pesar de que nos hemos limitado a escribir en un dialecto siendo tan diferentes los modos de decir de unos pueblos a otros, es imposible determinarlos todos aquí, y por lo mismo hemos tenido que fijarnos en lo que es más generalmente usual y mejor conocido en los varios distritos de esta provincia (Aranzábal 1883: 13). 
En la columna del español, los sustantivos aparecen tanto en singular como en plural, en masculino y femenino, y con artículo (definido o indefinido) o sin él. La asistematicidad en la elaboración de este repertorio queda reflejada igualmente en la presencia de entradas repetidas en diversos grupos (por ejemplo, la liebre aparece en «Cosas de comer y beber» y en «Los animales»), así como en voces colocadas en lugares en los que no deberían estar (arrepentirse, por ejemplo, se inserta dentro del grupo de verbos que se relacionan con las «Acciones que expresan obras o trabajos de mano»).

En la tabla siguiente se presentan los diferentes epígrafes en los que está dividido el vocabulario, junto con el número de entradas dentro de cada uno de ellos:

\begin{tabular}{|c|c|}
\hline EPÍGRAFES & ENTRADAS \\
\hline \multicolumn{2}{|l|}{ Nombre sustantivo } \\
\hline$\left[\right.$ Cosas que se consideran en el universo o mundo] ${ }^{11}$ & 6 \\
\hline [Cosas que pertenecen al globo terrestre o a la tierra] & 9 \\
\hline$[$ El agua $]$ & 3 \\
\hline [Cosas relativas a la atmósfera; el aire, los meteoros] & 17 \\
\hline$[$ Del tiempo] & 2 \\
\hline Partes del cuerpo humano & 48 \\
\hline Los sentidos & 10 \\
\hline Parentescos & 26 \\
\hline Cosas de comer y beber & 71 \\
\hline Prendas de vestir & 20 \\
\hline Cosas que pertenecen a los pueblos y casas & 43 \\
\hline Los animales & 55 \\
\hline Días de la semana & 7 \\
\hline Meses, estaciones y festividades del año & 23 \\
\hline \multicolumn{2}{|l|}{ Nombre adjetivo } \\
\hline Nombres adjetivos & 58 \\
\hline Grados del adjetivo & 28 \\
\hline Adjetivos numerales, absolutos o cardinales & 41 \\
\hline Adjetivos numerales ordinales & 40 \\
\hline \multicolumn{2}{|l|}{ Pronombre } \\
\hline Pronombres personales & 7 \\
\hline Pronombres demostrativos & 6 \\
\hline Pronombres posesivos & 6 \\
\hline Pronombres relativos & 4 \\
\hline Pronombres indeterminados & 2 \\
\hline \multicolumn{2}{|l|}{ Verbo } \\
\hline [Acciones del hombre] & 11 \\
\hline [Acciones relativas a la facultad de hablar] & 11 \\
\hline
\end{tabular}

${ }^{11}$ En los casos en los que no hay epígrafe, pongo los de la nomenclatura de Eguren, puesto que es la obra de la cual Aranzábal copia sus artículos. 


\begin{tabular}{|l|r|}
\hline [Acciones relativas a la vida] & 9 \\
\hline [Acciones relativas a los alimentos, al vestido y al descanso] & 8 \\
\hline [Acciones relativas al estudio y el entendimiento] & 11 \\
\hline [Acciones que manifiestan amor y odio] & 6 \\
\hline [Acciones relativas a los juegos de recreo y ejercicios] & 3 \\
\hline [Acciones que expresan movimiento] & 11 \\
\hline [Acciones que expresan obras o trabajos de mano] & 16 \\
\hline [Acciones que se refieren a las operaciones de compra y venta] & 11 \\
\hline [Acciones que se refieren al culto divino] & 3 \\
\hline Participios & 5 \\
\hline Participios activos & 10 \\
\hline Participios pasivos regulares & 10 \\
\hline Participios pasivos irregulares & \\
\hline Adverbios & 18 \\
\hline Adverbios de lugar & 19 \\
\hline Adverbios de tiempo & 11 \\
\hline Adverbios de modo & 11 \\
\hline Adverbios de cantidad & 3 \\
\hline Adverbios de orden & 4 \\
\hline Adverbios de afirmación & 4 \\
\hline Adverbios de negación & 1 \\
\hline Adverbios de duda & 60 \\
\hline Preposiciones & 2 \\
\hline Conjunciones & 1 \\
\hline Conjunciones copulativas & 3 \\
\hline Conjunciones disyuntivas & \\
\hline Conjunciones adversativas & \\
\hline Conjunciones condicionales & \\
\hline & \\
\hline & \\
\hline
\end{tabular}

Como puede observarse, la nomenclatura de Aranzábal no es muy extensa y presenta los epígrafes más habituales de este tipo de diccionarios. Los enunciados de la sección de los sustantivos son muy similares a los que encontramos en Eguren, pero su orden, que sigue el modelo teocéntrico ${ }^{12}$, puesto que empieza por Dios y los elementos del Universo, se asemeja más al utilizado por autores como Astigarraga o Jáuregui, que mantienen la disposición de Chantreau. Sin embargo, las entradas contenidas en dichos epígrafes están copiadas casi en su totalidad de la obra de Eguren, de forma resumida. Como ejemplo de ello, se presenta un conjunto de voces de «Partes del cuerpo humano» de Eguren y las correspondientes de la nomenclatura de Aranzábal:

${ }^{12}$ Ayala Castro (1992: 437) distingue el «orden teocéntrico medieval, comenzando por Dios, las divinidades, el Universo, y terminando con la muerte» y el «orden antropocéntrico renacentista con el hombre como punto de partida, las partes del cuerpo humano, los vestidos, etc., y las fiestas y juegos como temas finales». 


\begin{tabular}{|l|l|}
\hline \multicolumn{1}{|c|}{ EGUREN (186713) } & \multicolumn{1}{|c|}{ ARANZÁBAL (1883) } \\
\hline el cuerpo. & el cuerpo. \\
la cabeza. & la cabeza. \\
la frente. & la frente. \\
el cabello. & el cabello. \\
la barba (pelo). & la barba (pelo). \\
la barba. & \\
el bigote. & la cara. \\
la cara. & \\
el cerebro. & los sesos. \\
las sienes. & los ojos. \\
los sesos. & las pestañas. \\
los ojos. & \\
las pestañas. & \\
las cejas. & \\
los párpados. & la nariz. \\
las mejillas. & el labio. \\
la nariz. & la boca. \\
el labio. & \\
la boca. & \\
\hline
\end{tabular}

Otra prueba de ello son las numerosas entradas que Aranzábal introduce en su nomenclatura y que no están presentes en los repertorios vasco-románicos de Astigarraga o Jáuregui, y tampoco en Chantreau, pero sí en el de Eguren. Hallamos, por ejemplo, voces como las referidas los tipos de viento (el viento frio, el viento Norte, el viento Sur, el vendaval, el huracán) que solo se encuentran insertas en Eguren, dentro de «Cosas relativas a la atmósfera; el aire, los meteoros». Lo mismo ocurre también en otros epígrafes, como en «Los sentidos», en el que Aranzábal incluye las entradas la mirada y el ruido, que solo aparecen en Eguren; en «Parentescos», en el que se ofrecen entradas como los padres y la nodriza; o en «Partes del cuerpo humano», con las pestañas, los humores y el sudor.

No obstante, dentro del enunciado «Cosas que pertenecen a los pueblos y casas», se observa que Aranzábal ha podido seguir primeramente la nomenclatura de Eguren, pero que después ha añadido otras entradas, que coinciden con las del repertorio de Astigarraga y Ugarte. Entre estas últimas, vuelve a introducir tres voces seguidas que aparecen en la nomenclatura de Eguren. Para mostrar este hecho, en la tabla siguiente se han copiado las entradas de la obra de Eugenio de Aranzábal, y a ambos lados, las entradas de las nomenclaturas de Luis de Astigarraga y Ugarte y Juan María de Eguren:

\footnotetext{
${ }^{13}$ Como ya se ha comentado, utilizamos la edición de 1867 y no la de 1876 porque es más probable que haya sido esta la usada por Aranzábal para su obra. Como una prueba nueva de esto, hemos hallado varias entradas que se encuentran en la nomenclatura de Eguren de 1867 y que fueron eliminadas en la segunda edición de 1876, pero que sí aparecen en el repertorio de Aranzábal: los costados en «Partes del cuerpo humano», caldo limpio en "Cosas de comer y beber», ayunar, aprender de memoria, empezar o empezar, dentro de los verbos. Estas voces, además, están recogidas habitualmente en el mismo orden que las de Eguren en 1867.
} 


\begin{tabular}{|c|c|c|}
\hline ASTIGARRAGA (1825) & ARANZÁBAL (1883) & EGUREN (1867) \\
\hline 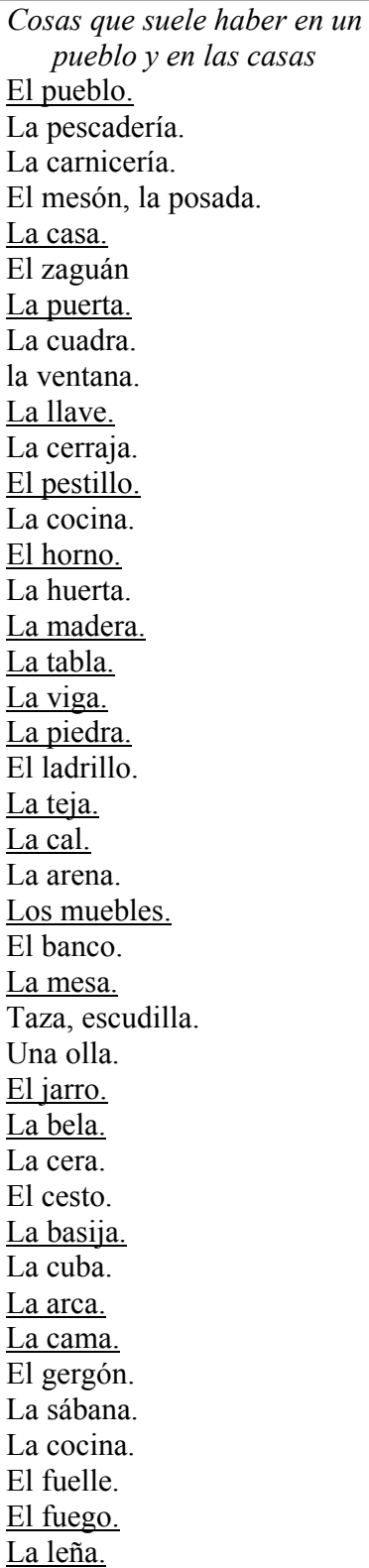 & 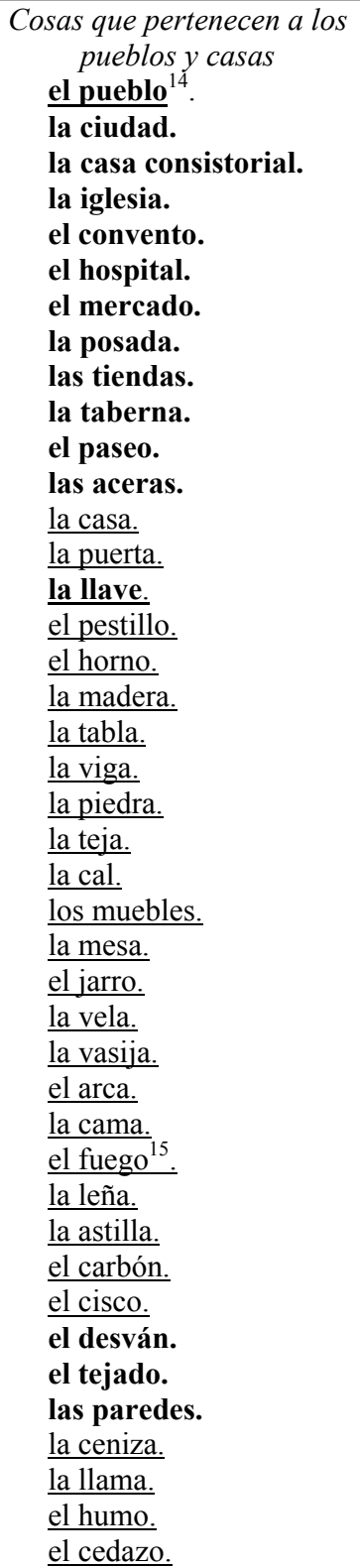 & $\begin{array}{l}\text { Cosas que pertenecen a los } \\
\text { pueblos y a las casas } \\
\text { el pueblo. } \\
\text { la ciudad. } \\
\text { el puerto de mar. } \\
\text { el muelle. } \\
\text { el embarcadero. } \\
\text { las calles. } \\
\text { la plaza. } \\
\text { la plazuela. } \\
\text { el callejón. } \\
\text { las casas. } \\
\text { la casa consistorial. } \\
\text { el castillo. } \\
\text { la Iglesia. } \\
\text { el teatro. } \\
\text { la carnicería. } \\
\text { el matadero. } \\
\text { la pescadería. } \\
\text { el convento. } \\
\text { el hospital. } \\
\text { el mercado. } \\
\text { la aduana. } \\
\text { el juzgado. } \\
\text { la fonda, la posada. } \\
\text { las tiendas. } \\
\text { un barrio. } \\
\text { la taberna. } \\
\text { el bodegón. } \\
\text { el paseo. } \\
\text { el correo. } \\
\text { la cárcel. } \\
\text { el palacio. } \\
\text { el colegio. } \\
\text { el empedrado. } \\
\text { las aceras. } \\
\text { la fuente. } \\
\text { el zaguçan. } \\
\text { el patio. } \\
\text { la vecindad. } \\
\text { los habitantes. } \\
\text { la casa de campo. } \\
\text { las puertas. } \\
\text { lantanas. } \\
\text { la }\end{array}$ \\
\hline
\end{tabular}

${ }^{14}$ Señalo en negrita las voces que coinciden en Aranzábal y Eguren; y subrayadas, las que Aranzábal copia de Astigarraga.

${ }^{15}$ Las entradas el fuego, la leña, el carbón, el cisco, la ceniza, la llama, el humo sí que se hallan en la nomenclatura de Eguren, pero dentro del epígrafe «El fuego, los combustibles». Asimismo, el libro se encuentra en el epígrafe «Cosas de la escuela» y el cedazo en «Menaje de cocina». 


\begin{tabular}{|l|l|l|}
\hline La astilla. & el libro. & el corral. \\
\hline El carbón. & la cuadra. \\
\hline El cisco. & la cochera. \\
\hline El tizón. & las habitaciones. \\
La ceniza. & la sala. \\
\hline La llama. & {$[\ldots]$} \\
El humo. & el desván. \\
El hollín. & el tejado. \\
La yesca. & la escalera. \\
El pedernal. & una reja. \\
La caldera. & las persianas. \\
El gancho. & el piso. \\
La errada. & la bóveda. \\
El asador. & el primer piso. \\
La cuerda, la soga. & el piso bajo. \\
El cujal. & las paredes. \\
El cedazo. & el pozo. \\
El libro. & la puerta falsa. \\
& el llamador, aldaba. \\
& & el picaporte. \\
\hline
\end{tabular}

Pocos sustantivos, por tanto, son nuevos en la nomenclatura de Aranzábal; tan solo pueden citarse algunas palabras que no he hallado dentro de otros repertorios vascorománicos, como chacolí y setas mayores ${ }^{16}$ en «Cosas de comer y beber» o la astilla («Cosas que pertenecen a los pueblos y a las casas»).

El capítulo perteneciente a los verbos tampoco es original, sino que es, de nuevo, una reproducción resumida de la nomenclatura de Eguren. A pesar de que las entradas se suceden sin que aparezca ningún epígrafe, se agrupan según los enunciados de este autor ${ }^{17}$ y siguen el mismo orden. Únicamente no aparecen en Eguren los verbos agarrar, engordar y arrepentirse. Véase el siguiente segmento de «Acciones del hombre» como muestra de la similitud entre las dos nomenclaturas:

\begin{tabular}{|l|l|}
\hline \multicolumn{1}{|c|}{ EGUREN (1867) } & \multicolumn{1}{c|}{ ARANZÁBAL (1883) } \\
\hline llorar. & llorar. \\
suspirar. & \\
bostezar. & toser. \\
toser. & rascarse. \\
rascarse. & silbar. \\
silvar. & oír. \\
oír. & \\
oler. & \\
sonarse. & sudar. \\
sudar. & \\
\hline
\end{tabular}

${ }^{16}$ En Eguren (1867) aparece, dentro de «Verduras y legumbres», «perrechicuac, las setas». Aranzábal, sin embargo diferencia entre «susac, las setas» y «perrechicuac, setas mayores».

${ }^{17} \mathrm{El}$ orden de los verbos en la nomenclatura de Chantreau es diferente, así como en la de Astigarraga y la de Jáuregui. 


\begin{tabular}{|l|l|}
\hline escupir. & escupir. \\
ver. & ver. \\
mirar. & mirar. \\
\hline
\end{tabular}

En cuanto a la sección de los adjetivos, parece que Aranzábal sigue la obra de Astigarraga, puesto que el contenido y el orden de los apartados son muy similares ${ }^{18}$, e incluso introduce algunas voces que aparecen en la nomenclatura de este autor y que no están presentes en el capítulo de adjetivos de Chantreau, obra de la cual se copia el repertorio (alguno, a; fulano, a; mucho, a; varios, as).

Aranzábal incluye aquí los numerales, tanto los ordinales como los cardinales, siguiendo el modelo de Astigarraga ${ }^{19}$, con una pequeña diferencia, ya que Astigarraga coloca los numerales cardinales dentro de los pronombres (los califica, sin embargo como «pronombres o adjetivos numerales»). Al final del listado de adjetivos, Aranzábal inserta un fragmento dedicado a los grados del adjetivo, lo que supone una innovación. Esta sección, probablemente por su contenido más gramatical que léxico, no aparece en ninguno de los repertorios temáticos vasco-románicos previos a Aranzábal que se conocen, y tampoco en la nomenclatura de Chantreau ${ }^{20}$ :

\begin{tabular}{|c|c|}
\hline Elcarraren maillac. & Grados del adjetivo. \\
\hline \multicolumn{2}{|c|}{ Positivo } \\
\hline Ona & bueno. \\
\hline \multicolumn{2}{|c|}{ Comparativo } \\
\hline $\begin{array}{l}\text { Aiñ ona } \\
\text { Obia }\end{array}$ & $\begin{array}{l}\tan \text { bueno. } \\
\text { mejor. }\end{array}$ \\
\hline \multicolumn{2}{|c|}{ Superlativo } \\
\hline Guztiz ona & muy bueno. \\
\hline \multicolumn{2}{|c|}{ Positivo } \\
\hline Ederra & hermoso \\
\hline \multicolumn{2}{|c|}{ Comparativo } \\
\hline $\begin{array}{l}\text { Aiñ ederra } \\
\text { Ederragoa }\end{array}$ & $\begin{array}{l}\text { tan hermoso. } \\
\text { más hermoso. }\end{array}$ \\
\hline \multicolumn{2}{|c|}{ Superlativo } \\
\hline Gustiz ederra & muy hermoso. \\
\hline
\end{tabular}

Tras los adjetivos, Aranzábal presenta los pronombres divididos en cinco grupos: personales, demostrativos, posesivos, relativos e indeterminados ${ }^{21}$. Como también ocu-

${ }^{18}$ Astigarraga copia su nomenclatura de la de Chantreau, pero sin embargo coloca los colores al comienzo del listado de adjetivos, orden que también encontramos en la obra de Aranzábal.

${ }^{19}$ Eguren, sin embargo, coloca los numerales ordinales y cardinales dentro de los sustantivos.

${ }^{20}$ Los grados del adjetivo sí están presentes en el Arte de Chantreau (1781: 70-73), pero no dentro de la nomenclatura.

${ }^{21}$ Astigarraga, en cambio, distingue los personales, los demostrativos, los posesivos, los relativos y los numerales, pero no los indeterminados. En la primera Gramática de la lengua castellana de la Academia (1771: 34-49) se diferencian los pronombres personales, demostrativos, posesivos y relativos; no obstante, dentro de este último apartado denomina alguien, alguno, ninguno y nadie como indefinidos. En la Gramática de la Academia de 1854 aparece el epígrafe de «Pronombres indeterminados», así como en el Compendio de la Gramática de la lengua castellana de 1857. 
rre en los adjetivos, en la columna castellana se introducen en la misma entrada las variantes en género masculino, femenino y neutro (en los casos en los que es posible), ya que el euskera no hace tal diferencia.

\begin{tabular}{|l|l|}
\hline Icurasteco icen ordecoac & Pronombres demostrativos \\
Au & este, esta, esto, \\
Ori & ese, esa, eso. \\
Bestia & aquel, aquella, o. \\
Onec & estos, estas. \\
Orrec & esos, esas. \\
Bestiac & aquellos, aquellas. \\
\hline
\end{tabular}

Tras los verbos, se insertan los participios, clasificados en participios activos, participios pasivos regulares y participios pasivos irregulares ${ }^{22}$. Los adverbios se distinguen asimismo en adverbios de lugar, de tiempo, de modo, de cantidad, de orden, de afirmación, de negación y de duda ${ }^{23}$.

Las preposiciones se presentan alfabéticamente a partir de las preposiciones castellanas. Dentro de cada uno de los apartados, se ofrecen varias entradas con diferentes tipos de palabras (nombres propios y comunes, palabras acabadas en vocal o en consonante) porque en euskera las terminaciones varían según estos parámetros. Además, una preposición castellana puede traducirse en la lengua vasca mediante varias posposiciones, según el autor. Un ejemplo de esto son las entradas de las preposiciones $a$ y de:

\begin{tabular}{|l|l|}
\hline \multicolumn{1}{|c|}{ LEIPINTZAC } & \multicolumn{1}{c|}{ PREPOSICIONES } \\
\hline Ri, $i$, ari, ei, ra, ca. & \multicolumn{1}{c|}{$a$} \\
Franciscori & a Francisco. \\
Martin i & a Martín. \\
Lurrari & a la tierra. \\
Lurr ei & a las tierras. \\
Echera & a casa. \\
Arrica & a pedradas. \\
& \\
Ren, rena, arena, coa, goa, dic, tic. & \multicolumn{1}{c}{ De. } \\
Justoren chacurra & el perro de Justo. \\
Pedro rena & de Pedro. \\
Guizon aren gorputza & el cuerpo del hombre. \\
Durango coa & de Durango. \\
Jemein goa & de Jeméin. \\
Nun goa & de dónde. \\
Nun dic & de dónde. \\
Eche tic & de casa. \\
\hline
\end{tabular}

\footnotetext{
${ }^{22}$ Astigarraga, en cambio, únicamente diferencia entre participios irregulares y regulares.

${ }^{23}$ La misma clasificación que aparece ya en la primera Gramática de la Academia (1771: 189-191).
} 
Por último, Aranzábal introduce las conjunciones, divididas en copulativas $(y, e, n i$, $q u e$ ), disyuntivas $(o, u)$, adversativas (pero, mas, sino, aunque, cuando) y condicionales (si, como).

Al final de la primera parte, dado el carácter práctico de la obra, Aranzábal introduce una serie de ejercicios para que los niños trabajen lo aprendido hasta ese momento. Los ejercicios se ordenan de menor a mayor dificultad y según lo que se ha ido estudiando: primeramente, se presentan segmentos formados por un sustantivo y un adjetivo de grado positivo (oveja negra, libro nuevo, casa grande, pueblo pequeño); después, un sustantivo y un adjetivo en grado comparativo o superlativo (jarra más llena, mejor cosa, muy buen pueblo) o un numeral (dos escuelas, veinte niños, segunda vez); tras esto, construcciones con pronombres y adverbios (ese mejor que aquel, estos ayer, aquellos muy malos); o de verbo más un complemento, ya sea un sintagma nominal o adverbial (mirar bien, decir la verdad, responder despacio, comer el pan); y, finalmente, Aranzábal ofrece construcciones con un verbo y varios complementos (atar lejos el novillo, luego venir acá, ser siempre bien visto).

\section{CONCLUSIÓN}

El Método de Eugenio de Aranzábal tiene como finalidad la enseñanza del español a los alumnos de las escuelas vizcaínas situadas en entornos rurales. En estos lugares, a finales del siglo XIX la lengua más utilizada seguía siendo el euskera, por lo que los niños tenían dificultades para poder adquirir los conocimientos impartidos en lengua castellana. Aranzábal, conocedor de este problema, elabora una obra con la que los niños adquieran de una manera rápida y eficaz la lengua española, en la que inserta un breve vocabulario ordenado temáticamente.

Esta nomenclatura no es un repertorio original, sino que reproduce lo realizado por autores vascos precedentes. Aranzábal copia y resume buena parte del método utilizado anteriormente por Juan María de Eguren, tanto en la estructura como el contenido, método que había cosechado un gran éxito. Se ha hallado también una gran similitud con la obra de Astigarraga y Ugarte, que proviene a su vez del Arte de Chantreau. No encontramos, por tanto, un gran número de novedades en los epígrafes presentados ni en las entradas de cada uno de ellos, con excepción de varias voces y algún apartado, como el de los grados de los adjetivos, que, en realidad, es más de contenido gramatical que léxico.

No obstante, este texto es una muestra de los métodos utilizados en el siglo XIX para la enseñanza de la lengua española y su implantación en las escuelas del País Vasco, uno de los territorios bilingües de la Península. Es también uno de los escasos ejemplos de nomenclaturas con la lengua vasca, por lo que es de gran importancia para el estudio de la lexicografía vasco-románica y para un mayor conocimiento de la historia de los diccionarios del español. 


\section{REFERENCIAS BIBLIOGRÁFICAS}

\section{FUENTES PRIMARIAS}

ARANZÁBAL, Eugenio de (1883): Método práctico de enseñar el castellano a los niños vascongados en las escuelas de Vizcaya, Bilbao, Librería de Segundo Salvador. Ejemplar de la Biblioteca Nacional de España, Madrid, 1/64079.

Astigarraga Y UGARTE, Luis de (1825): Diccionario manual bascongado y castellano, y elementos de gramática. Para el uso de la juventud de la M. N. y M. L. Provincia de Guipúzcoa, con ejemplos y parte de la Doctrina Cristiana en ambos idiomas, San Sebastián, Imprenta de Ignacio Ramón Baroja. Ejemplar de la Biblioteca Koldo Mitxelena, San Sebastián, J. U. 4265, <http://irudi.gipuzkoa.net/WAS/CORP/DCMVisorBibliotecaWEB/

/Visor.do?id=/Bi_Biblioteca[@PH_Amicus=\%2217748\%22]\&hizkuntza=C $>$ [última consulta: 16/07/2014].

(1827): Diccionario manual bascongado y castellano, y elementos de gramática.

Para el uso de la juventud de la M. N. y M. L. Provincia de Guipúzcoa, con ejemplos y parte de la Doctrina Cristiana en ambos idiomas, Tolosa, Imprenta de D. Juan Manuel de la Lama. Ejemplar de la Biblioteca Nacional de España, Madrid, U/1395, http://bdhrd.bne.es/viewer.vm?id=0000039770\&page=1 [última consulta: 16/07/2014].

CHANTREAU, Pierre Nicolás (1781): Arte de hablar bien francés o gramática completa dividida en tres partes, Antonio de Sancha, Madrid. Ejemplar de la Biblioteca de Catalunya, Barcelona, R(4)-8-31, <http://books.google.es/books?id=UFCVPzgeCRMC\&printsec=frontcover

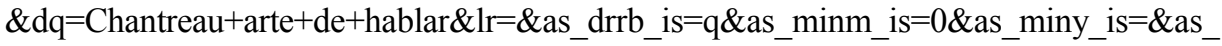
maxm_is $=0 \& a s \_m a x y \_$is $=\& a s \_b r r=1 \& c \bar{d}=3>$ [última consulta: $\left.16 / 07 / 2014\right]$.

EGUREN, Juan María de (1867): Método práctico de enseñar el castellano en las escuelas vascongadas, Vitoria, Imprenta del Semanario Católico Vasco-Navarro. Ejemplar de la Bibliothèque Nationale de France, París, Z BASQUE-280, $<$ http://gallica.bnf.fr/ark:/12148/bpt6k9353187> [última consulta: 16/07/2014].

(1868): Diccionario manual o vocabulario vasco-castellano para el uso de los vascongados que quieran aprender el castellano, Vitoria, Imprenta de El semanario Católico Vasco-Navarro. Ejemplar de la Fundación Sancho El Sabio, Vitoria-Gasteiz (signatura: ATA 1549), <http://hdl.handle.net/10357/1382> [última consulta: 16/07/2014].

- (1876): Diccionario vasco-castellano y método para enseñar el castellano a los vascongados, San Sebastián, Imprenta de Baroja. Ejemplar de la Fundación Sancho El Sabio, Vitoria-Gasteiz, ATV 9138, <http://hdl.handle.net/10357/1702> [última consulta: $16 / 07 / 2014]$.

REAL ACADEMIA ESPAÑOla (1771): Gramática de la lengua castellana, Madrid, Joaquín de Ibarra. Ejemplar de la Biblioteca Nacional de España, Madrid, R/38876, < http://bdhrd.bne.es/viewer.vm?id=0000063358\&page=1 $>$ [última consulta: 16/07/2014].

- (1854): Gramática de la lengua castellana, Madrid, Imprenta Nacional. Ejemplar de la Biblioteca Nacional de España, Madrid, 1/63262, < http://bdhrd.bne.es/viewer.vm?id=0000057873\&page=1> [última consulta: 16/07/2014].

- (1857): Compendio de la Gramática de la lengua castellana dispuesto por la Real Academia Española, para la Segunda Enseñanza, Madrid, Imprenta Nacional. Ejemplar de la Universidad Complutense de Madrid, Madrid, FH 2896, $<$ http://books.google.es/books/ucm?vid=UCM5326067544\&printsec=frontcover\&redir _esc $=y>$ [última consulta: 16/07/2014]. 


\section{FUENTES SECUNDARIAS}

ACERo DURÁNTEZ, Isabel (2003): «La lexicografía plurilingüe del español», en Antonia M. ${ }^{a}$ Medina Guerra, coord., Lexicografía española, Barcelona, Ariel, pp. 175-204.

AlVAR EZQUERRA, Manuel (1987): «Apuntes para la historia de las nomenclaturas del español», en Actas del VII Congreso Internacional de Lingüistica y Filología de América Latina (ALFAL). Homenaje a Pedro Henríquez Ureña, Santo Domingo, Universidad Nacional Pedro Henríquez Ureña, pp. 457-470.

(2002): De antiguos y nuevos diccionarios del español, Madrid, Arco Libros. (2011): Las nomenclaturas del español. Siglos XV-XIX, Madrid, Liceus.

Ayala CASTRO, M. Concepción (1992): «El concepto de nomenclatura», en Manuel Alvar Ezquerra, ed., Actas del IV Congreso Internacional Euralex '90, Barcelona, Biblograf, pp. 437-444.

AZORÍN FERNÁNDEZ, Dolores (2000): Los diccionarios del español en su perspectiva histórica, Alicante, Universidad de Alicante.

Cundín SANTOS, Margarita y María Ángeles LíBANO ZuMALACÁRREGUI (2000): «La lexicografía bilingüe euskera-castellano, castellano-euskera», en Ignacio Ahumada Lara, ed., Cinco siglos de lexicografía del español: IV Seminario de Lexicografía Hispánica, Jaén, Universidad de Jaén, pp. 307-339.

EChENIQUe ElizONDO, M. ${ }^{a}$ Teresa $\left(1987^{2}\right)$ : Historia lingüística vasco-románica, Madrid, Paraninfo.

(1997): Estudios lingüísticos vasco-románicos, Madrid, Istmo.

$\left(2005^{2}\right)$, «La lengua vasca en la historia lingüística hispánica», en Rafael Cano, coord., Historia de la lengua española, Barcelona, Ariel, pp. 59-80.

GARCÍA ARANDA, M. ${ }^{a}$ Ángeles (2009): «La enseñanza del español en las escuelas del País Vasco: la labor lexicográfica de Juan María de Eguren (1867-1876)», Boletín de Filología de la Universidad de Chile, XLIV, 1, pp. 97-124.

(2010): «Nomenclaturas decimonónicas del español», Boletín de Lingüistica, 33, pp. 5-28.

(2011a): «La influencia del Arte de hablar bien francés de P. N. Chantreau (1781) en la lexicografía vasca decimonónica», en M. C. Ayala Castro, coord., La producción lexicográfica: investigación y análisis, Málaga, Universidad de Málaga, pp. 81-105.

(2011b): «La evolución de la lexicografía vasco-románica: las obras de Astigarraga, Jáuregui, Eguren y Juvenal Martyr», Oihenart, 26, pp. 197-215.

GÓMEZ, Ricardo (2008): «Las ideas lingüísticas vascas en los siglos XVII-XIX», presentado en el seminario Monumenta Linguae Vasconum Proiektua (2002-2008). Sei urte ikerketan, Universidad del País Vasco, <http://www.ehu.es/monumenta/pdf/mintegia20 08/Gomez-Ideas linguisticas.pdf $>$ [última consulta: 02/07/2014].

MichelenA, Luis (1974 [2011]): «De lexicografía vasca. A propósito de Axular-en hiztegia del Padre Luis Villasante», Fontes Linguae Vasconum, 6, pp. 103-121. En Joseba A. Lakarra e Iñigo Ruiz Arzalluz, eds., Obras completas VIII: Lexicografia, Historia del léxico, Etimología, San Sebastián y Vitoria, Universidad del País Vasco.

MINISTERIO DE FOMENTO (1887): Colección legislativa de primera enseñanza, Madrid, Imprenta del Colegio Nacional de Sordo-Mudos y de Ciegos. Edición digital de la Biblioteca Virtual Miguel de Cervantes, 1999, <http://www.cervantesvirtual.com/nd/ark:/59851/ bmc0v8b6> [última consulta: 07/07/2014]. 